\title{
Metallographische Mitteilungen aus dem Institut fur anorganische Chemie der Universität Göttingen.
}

\section{XXIX.}

\section{Über das Zustandsdiagramm von Eisen und Schwefel. \\ Von}

W. Treitsohke und G. Tammann.

Mit 2 Figuren im Text und 1 Tafel.

In Gmernr-KraUts Handbuch sowie in der erst kürzlich erschienenen Chimie minerale von H. Morssan sind die folgenden 7 Eisen-Schwefelverbindungen

$$
\mathrm{FeS}_{2}, \mathrm{Fe}_{2} \mathrm{~S}_{3}, \mathrm{Fe}_{7} \mathrm{~S}_{8}, \mathrm{FeS}, \mathrm{Fe}_{4} \mathrm{~S}_{3}, \mathrm{Fe}_{2} \mathrm{~S}, \mathrm{Fe}_{8} \mathrm{~S}, \mathrm{Fe}_{11} \mathrm{~S}_{12}
$$

aufgefuhrt. H. le Chateinrer und Zregler ${ }^{1}$ haben durch eine mikroskopische Untersuchung gezeigt, dafs die Reguli, die man durch Zusammenschmelzen von Eisen und Schwefeleisen orhält, Gemenge von Eisen- und Schwefeleisenkristallen sind. Da dasselbe auch von den Konglomeraten der Gesamtzusammensetzung $\mathrm{Fe}_{4} \mathrm{~S}_{3}, \mathrm{Fe}_{2} \mathrm{~S}$ und $\mathrm{Fe}_{8} \mathrm{~S}$ gilt, so ist damit erwiesen, dafs diese vermeintlichen Verbindungen nicht existieren. Durch die Arbeit ron H. le ChateLIFR und ZIEGLER ist eine Hauptfrage, welche das System Eisen und Schwefeleisen betrifft, erledigt. Betreffs, des Zustandsdiagrammes der Gemenge von Eisen und Schwefeleisen liegen nur spärliche Angaben vor. H. le Chatelier und Zieguer konstatieren vor allem eine äufserst interessante Umwandlung des Schwefeleisens bei $130^{\circ}$. Ferner mufste man aus der Struktur der von H. le Chatreurks und ZiraLrer mikroskopisch untersuchten Gemenge von Eisen und Schwefeleisen schliefsen, dafs die Löslichkeit des Eisens bei dem Schmelzpunkt des FeS nicht erheblich ist und dafs dieselbe mit

1 Balletin de la Société d'Encouragement pour l'Industrie, T. 101 , p. 368, 1902. 
steigender Temperatur schnell zunimmt, denn in den Eisen-Schwefeleisenkonglomeraten enthält das aus $\mathrm{Fe}$ und $\mathrm{FeS}$ bestehende Eutektikum nur geringe Mengen von Eisen. Le Chateunfer hat über das Schmelzdiagramm von FeS-Fe, indem er sich in diesem Punkte die gröfste Reserve auferlegt, keine Ansicht ausgesprochen. In der Tat liegen die Dinge wesentlich anders als man auf Grundlage des mikroskopischen Befundes, der auf die Mischbarkeit von FeS und $\mathrm{Fe}$ in allen Verhältnissen und die Kristallisation der beiden Komponenten in reinem Zustande deutet, annehmen könnte. Es hat sich nämlich bei genauerer Untersuchung ergeben, dafs sich flüssiges Eisen und Schwefeleisen nicht in allen Verhältnissen miteinander mischen. Diese Tatsache ist um so überraschender, weil man keine deutliche Schichtenbildung in den Systemen aus $\mathrm{FeS}$ und $\mathrm{Fe}$ beobachtet hat, und doch wäre bei der nicht unerheblichen Differenz in der Dichte der beiden Schichten eine schnelle Sonderung derselben vor Beginn der Kristallisation des Eisens zu erwarten; der Grund für das Nichteintreten derselben ist in der hohen Viskosität des flüssigen, mit Eisen gesättigten Schwefeleisens zu suchen.

Das Zustandsdiagramm der Fe-FeS-Mischungen ist also durch die Abwesenheit irgendeiner Schwefeleisenverbindung zwischen den Komponenten $\mathrm{Fe}$ und $\mathrm{FeS}$, sowie durch die Nichtmischbarkeit von flüssigem Eisen und flüssigem FeS beim Schmelzpunkt des Eisens charakterisiert, aufserdem treten noch die bekannten Umwandlungen des Eisens und die von H. le Chatelier und Zinglaer gefundene Umwandlung des Schwefeleisens bei $130^{\circ}$ ein, und aus den Schmelzen scheiden sich nicht die reinen Komponenten, sondern Mischkristalle aus.

\section{Die Versuche.}

Die flüssigen Mischungen von Eisen und FeS wurden durch Zusammenschmelzen von einem reinen Flufseisen, dessen Analyse früher mitgeteilt wurde ${ }^{1}$, und käuflichem Schwefeleisen hergestellt. Die Analyse dieses Schwefeleisens ergab im Mittel aus drei Sehwefelbestimmungen $28.25 \% \pm 0.05 \%$ Schwefel und $69.33 \%$ Eisen. Der Formel FeS würden $36.46 \% \mathrm{~S}$ und $63.54 \% \mathrm{Fe}$ entsprechen. Auf der Schlifffläche dieses Schwefeleisens (Fig. 1, Taf. I) sieht man in einer Grundmasse von $\mathrm{FeS}$ zahlreiche helle Eisenkörner und aufserdem noch ein eutektisches Strukturelement, von dem Le Chatrumer

1 Z. anorg. Chem. 47 (1905), 165. 
und ZrEGLER nachgewiesen haben, dals es aus einem Eisenoxyd und Schwefeleisen besteht, denn durch Erhitzen im Wasserstoffstrom verschwindet dies Strukturelement und beim Erhitzen in einer sauerstoffhaltigen Atmosphäre vermehrt sich die Menge desselben. Auf Grundlage obiger Analyse des Schwefeleisens wurde seine quantitative Zusammensetzung in folgender Weise berechnet. Es wurde angenommen, dafs aller Schwefel mit Eisen verbunden ist, und dafs der Rest, $100 \%$ - (Schwefel + Eisen) aus Sauerstoff besteht. Ferner nahmen wir in Ermangelung sicherer Kenntnisse über die Natur des dem käuflichen Schwefeleisen beigemengten Oxydes an, dafs dasselbe der Formel $\mathrm{Fe}_{3} \mathrm{O}_{4}$ entspreche und reduzierten die dann sich ergebenden Mengen von $\mathrm{Fe}$ und $\mathrm{FeS}$ auf 100. Die unter diesen Annahmen berechnete Zusammensetzung des käuflichen Schwefeleisens und der mit demselben dargestellten Schmelzen findet man in Tabelle 1. Wie man sieht, ist der Gehalt an Oxyden in den eisen. reichen Schmelzen zu vernachlässigen, von den Schmelzen mittlerer Konzentration an aber wächst derselbe doch recht erheblich. Trotzdem hielten wir es nicht für notwendig, schon jetzt die Untersuchung der Schmelzen mittlerer Konzentration unter Anwendung eines oxydfreien Schwefeleisens zu wiederholen, denn es stellte sich heraus, dafs Schwefeleisen Porzellan - besonders oberhalb $1400^{\circ}$ - schnell angreift. Die Auflösung des Porzellans geht so schnell vor sich, dafs die Porzellanschutzröbren des Thermoelementes, deren Wandstärke $1 \mathrm{~mm}$ betrug, bei $1600^{\circ}$ in den schwefeleisenreichen Schmelzen in weniger als einer Minute durchgefressen wurden. Magnesiaschutzrohre und Magnesiagefäfse konnten nicht angewandt werden, da sich in dieselben das Schwefeleisen kapillar einsog. Diese Umstände hinderten uns an der Darstellung von Schmelzen, welche nur Fe und $\mathbf{S}$ enthalten. Dazu kommt noch, dafs der Partialdruck des Schwefels in den schwefelreichen Schmelzen ein recht erheblicher ist, wodurch auch bei schnellem Schmelzen, besonders bei höheren Temperaturen, nicht unerhebliche Verluste an Schwefel eintreten können. Um eine Oxydation der Schmelzen zu vermeiden, wurden die Schmelzungen in einer Stickstoffatmosphäre vorgenommen.

Die Analyse der Schmelze, zu deren Herstellung 50\% Wisen und $50 \%$ käufliches Schwefeleisen eingewogen waren, zeigt, dafs nach einer Erhitzung auf $1540^{\circ}$ durch das Schmelzen ein Verlust von $2 \%$ Schwefel, entsprechend $5.7 \% \mathrm{FeS}$, stattgefunden hatte. In den eisenreichen Schmelzen wird diese Konzentrationsänderung durch das Schmelzen mit steigendem Fe-Gehalt abnehmen und in 
den eisenärmeren Schmelzen mit abnehmendem Eisengehalt zunehmen. Korrektionen, die sich auf die Zusammensetzung der Schmelzen nach ihrer Schmelzung beziehen, wurden nicht angebracht.

Zur Untersuchung der schwefelreichsten Mischungen wurde reines Eisenpulver mit etwas überschüssigem Schwefel gut gemengt und bei allmählichem Eintragen in ein schwer schmelzbares Glasrohr auf Rotglut erhitzt. Die so erhaltene Masse mufste nochmals in kleinen Portionen von etwa $5 \mathrm{~g}$ im Porzellanrohr zusammengeschmolzen werden, um ein Herausschleudern der Masse durch die sich entwickelnden Schwefeldämpfe zu verhindern. Nach dem Erhitzen auf $1400^{\circ}$ wurde eine Analyse dieser Schmelze ausgeführt, welche $35.05 \% \mathrm{~S}$ und $62.69 \%$ Eisen ergab; nimmt man an, dafs der Fehlbetrag von $2.26 \%$ aus gelöstem Porzellan besteht, so ergibt sich, dals die Masse $98.3 \% \mathrm{FeS}$ und $1.7 \%$ Fe enthielt.

Zur Darstellung der zweiten und dritten FeS-reichen Schmelzen wurde dieses reinere $\mathrm{FeS}$ mit Eisen gemengt, dann wurde geschmolzen, die Abkühlungskurven bestimmt und schliefslich die Reguli analysiert. Die eisenreichen Schmelzen - bis zu 50\% Eisen - wurden bis $1650^{\circ}$ und die folgenden auf $1500^{\circ}-1400^{\circ}$ erhitzt, darauf wurde mit einem Porzellanstabe im Stickstoffstrom gut umgeruhrt und schliefslich das Thermoelement, geschützt durch ein Porzellanrohr, in die Schmelze, deren Menge immer $20 \mathrm{~g}$ betrug, eingeführt und die Abkühlungskurve bestimmt. Die Temperaturen, bei denen Verzögerungen der Abkühlungsgeschwindigkeit auftreten, sind in folgender Tabelle, bezogen auf die Skala des Luftthermometers, verzeichnet. In dieser Tabelle findet man auch die Zeitdauer der Haltepunkte, $\Delta z$, multipliziert mit der Abkühlungsgeschwindigkeit $\frac{d t}{d} \frac{t}{z}$ bei der Temperatur des Haltepunktes. Diese Produkte, die auf gleiche Abkühlungsgeschwindigkeiten reduzierten Zeitdauern von Reaktionen in nonvarianten Systemen, sind proportional den Energiemengen, welche bei der Abkühlung durch jene Reaktion frei werden.

\section{Erklärung des Diagrammes.}

Das Zustandsdiagramm der Fe-FeS-Mischungen ist vor allem dadurch charakterisiert, dafs man bei zwei verschiedenen Temperaturen, $1400^{\circ}$ and $970^{\circ}$, Haltepunkte findet. Bei $1400^{\circ}$ erstrecken sich dieselben von $80 \%-8 \% \mathrm{FeS}$ und bei $970 \%$ von $97 \%-3 \% \mathrm{FeS}$. Diese Tatsachen schliefsen das Auftreten von Verbindungen aus und 


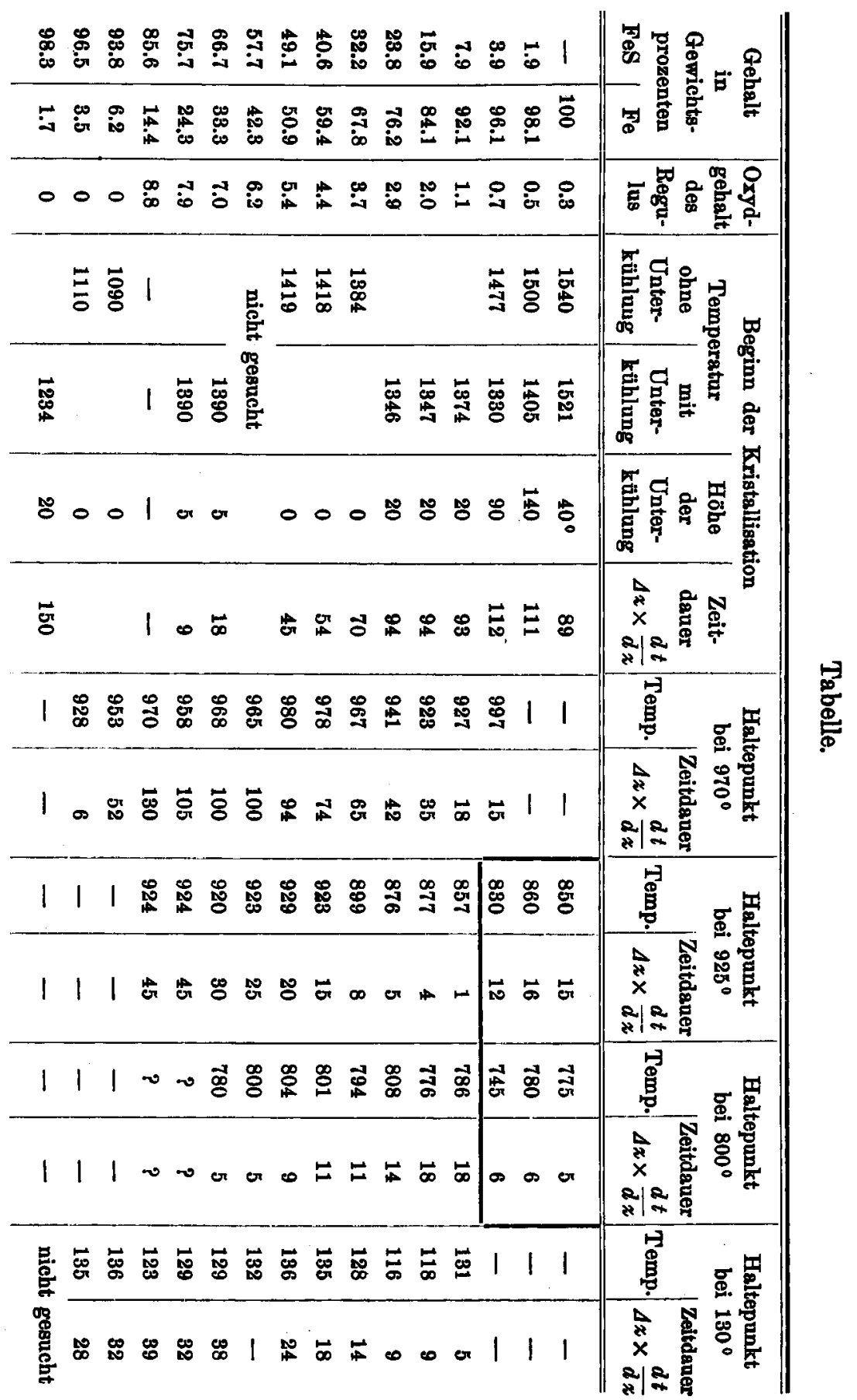


zeigen, dafs bei $1400^{\circ}$ eine Lösung von $20 \% \mathrm{Fe}$ im FeS mit einer Lösung von $8 \% \mathrm{FeS}$ in Eisen miteinander im Gleichgewicht sind.

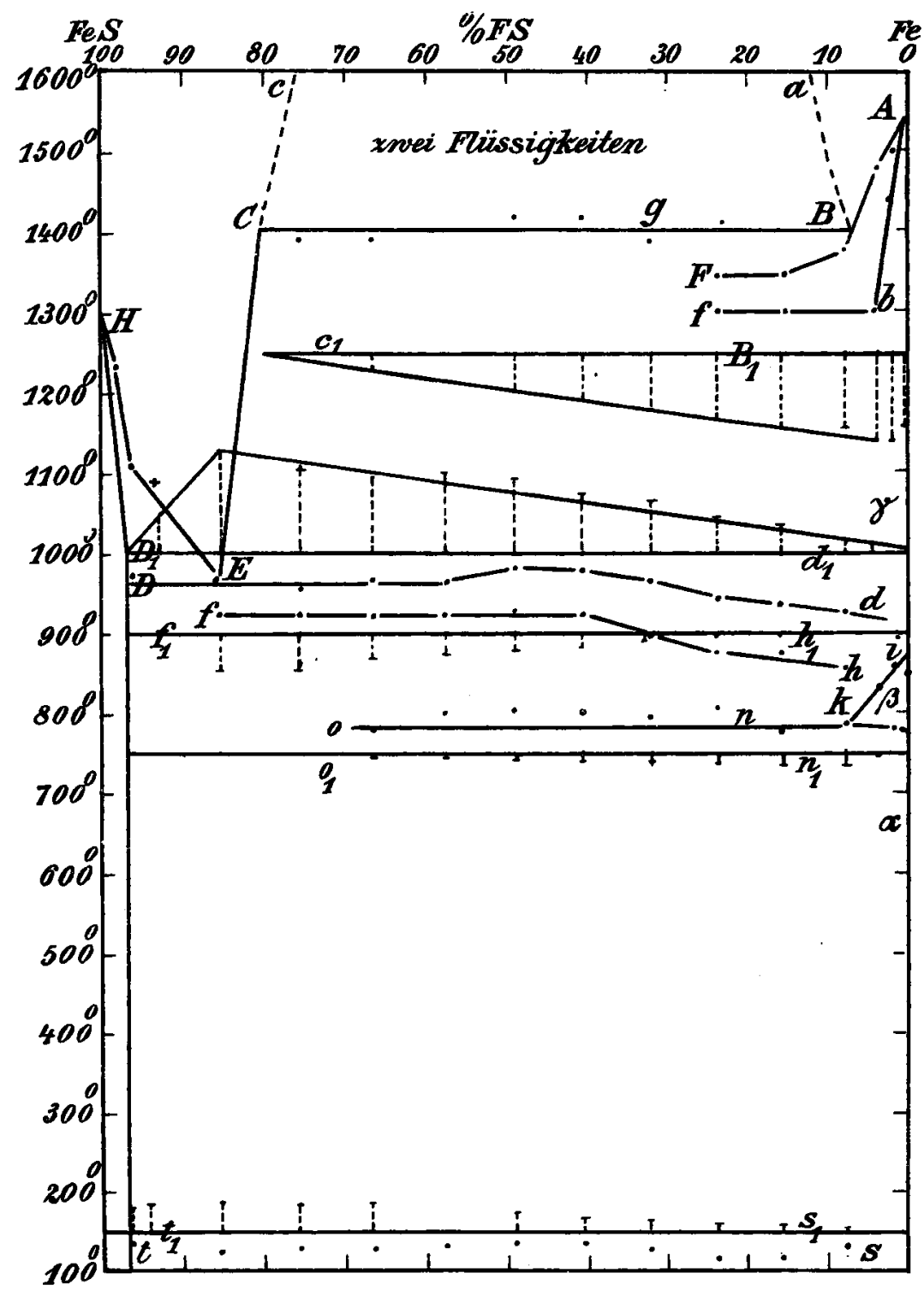

Fig. 1.

Flüssiges Eisen und Schwefeleisen mischen sich also beim Schmelzpunkt des Eisens nicht in allen Verhältnissen miteinander. Die Z. anorg. Chem. Bd. 49. 
beiden Kurven $a B$ und $c C$ deuten den Verlauf der Löslichkeit von $\mathrm{FeS}$ in $\mathrm{Fe}$ und umgekehrt an. Wie gewöhnlich konnten die beiden Kurven $c C$ und $a B$ nicht verfolgt werden, weil erstens die Wärmeentwickelung, welche die Entmischung begleitet, in der Regel eine sehr geringe ist, und weil zweitens solche Kurven meist sehr steil zu höheren Temperaturen ansteigen, bis sie sich einander zu krümmen und dann in einem Punkt zusammentreffen, der die böchste Temperatur des aus zwei flüssigen Phasen bestehenden Systemes angibt.

Bei der Kristallisation der eisenreichen Schmelzen tritt aber eine Komplikation ein. Bei der Geschwindigkeit, mit der die Schmelzen von $1600^{\circ}-1200^{\circ}$ abkühlten, etwa $2^{\circ}$ pro Sekunde, trat in den eisenreichen Schmelzen eine Ausscheidung von Mischkristallen ein, wie sich aus der Form der diesbezüglichen Abkühlungskurven ergab. Die Abkühlungskurve der Schmelze mit 97.5\% Fe unterscheidet sich von der des reinen Eisens sehr wenig, und auf den Abkühlungskurven der Schmelzen mit 90, 80 und $70 \%$ Fe treten sehr deutliche Kristallisationsintervalle auf, deren obere Temperaturen durch die Linie $F B$ und deren untere Temperaturen durch die Linie $f b$ im Diagramm bezeichnet sind. In diesem Konzentrationsgebiete tritt die Kristallisation immer erst nach einer vorausgegangenen Unterkühlung ein, deren Betrag einmal bis auf $140^{\circ}$ stieg. Um die Koordinaten der Kurve $A B$ zu bestimmen, mufsten die betreffenden Schmelzen mit Eisenstückchen geimpft werden.

Wächst nun der Gehalt an Schwefeleisen, so tritt die Kristallisation ohne Unterkühlung ein, und die durch dieselbe hervorgerufene Verzögerung der Abkühlung nimmt auf den Abkühlungskurven die Form eines Haltepunktes an. Gleichzeitig steigt die Temperatur dieser bei derselben Temperatur liegenden Haltepunkte um etwa $25^{\circ}$. Diese Haltepunkte liegen im Mittel bei $1400^{\circ}$ entsprechend der horizontalen Linie $C g$. Die Zeitdauer der Kristallisation bei $1400^{\circ}$, deren Werte auf der Horizontalen $c_{1} g_{1}$ aufgetragen sind, nimmt mit steigendem Gehalt an $\mathrm{FeS}$ ab und wird bei der Konzentration $C$ bei etwa $80 \% \mathrm{FeS}$ Null. Diese Konzentration entspricht sehr nahe der Zusammensetzung der einen flüssigen Phase $C$.

Die Zusammensetzung der eisenreichen Flüssigkeit, welche mit der Flüssigkeit $C$ bei $1400^{\circ}$ im Gleichgewicht sich befindet, kann nicht direkt bestimmt werden, da die Kristallisation der eisenreichen Schmelzen sich in einer ganz abnormen Weise vollzieht. Es bilden sich nämlich, wie aus den oben beschriebenen Abkühlungskurven 
dieser Schmelzen zu schliefsen ist, Mischkristalle, deren Zusammensetzung angenähert durch die Linie $A b$ angegeben wird. Man hat also anzunehmen, dafs sich aus den Schmelzen von $0-4 \% \mathrm{Fe}$ Mischkristalle von der Zusammensetzung der Schmelzen ausscheiden. Dieselben Mischkristalle scheiden sich auch aus den Schmelzen von 4-25\% FeS aus, aber während ihrer Abscheidung spaltet sich von der Fe-reichen Flüssigkeit noch die FeS-reiche Flüssigkeit $C$ ab.

Die Tatsachen, dals in den Schmelzen ron 8-25\% FeS die Kristallisation des gesättigten Mischkristalles $b$ sich innerhalb eines Temperaturintervalles vollzieht und dafs die Temperatur, bei der aus den Fe-ärmeren Schmelzen, von 80-30\% FeS, die Ausscheidung des gesättigten Mischkristalles $b$ vor sich geht, konstant ist und um etwa $50^{\circ}$ höher liegt als die Temperatur des Beginns der Kristallisation zwischen 25 und $75 \% \mathrm{FeS}$, möchten wir mit dem Gehalt der Schmelzen an Eisenoxyden in Zusammenhang bringen. Wenn diese in den Schmelzen von 80-30\% FeS sich hauptsächlich in der FeS-reichen Flüssigkeit befinden und wenn diese in den Schmelzen von $25-5 \% \mathrm{FeS}$ sich in der eisenreicheren Flüssigkeit anreichern, so wären jene Tatsachen verständlich.

Die FeS-reiche Schmelze, welche bei $1400^{\circ}$ mit der Fe-reichen Schmelze und den eisenreichen Mischkristallen $b$ im Gleichgewicht ist, enthält, wie wir sahen, $80 \% \mathrm{FeS}$, denn bei dieser Konzentration verschwinden die Haltepunkte bei $1400^{\circ}$. Andererseits hat die Zeitdauer der Haltepunkte bei $970^{\circ}$, welche durch gleichzeitige Kristallisation von $\mathrm{Fe}$ und $\mathrm{FeS}$ aus den Schmelzen zustande kommen, ihren maximalen Wert bei $85 \%$ FeS. Dieses ist also auch die Konzentration der eutektischen Schmelze $E$. Die Punkte $E$ und $C$ mufs die Löslichkeitskurve von $\mathrm{Fe}$ in FeS verbinden. Da ein Punkt auf der Kurve zufällig nicht bestimmt worden ist, sind die beiden Punkte $E$ und $C$ durch eine Gerade verbunden worden. Vom Punkte $E$ aus steigt eine zweite Linie $\mathbb{E H} \mathrm{zu}$ höheren Temperaturen; diese gibt den Beginn der Kristallisation von $\mathrm{FeS}$ an. Zur Bestimmung der Punkte dieser Kurve mufste von oxydfreiem FeS, das aus $\mathbf{S}$ und Fe dargestellt wurde, ausgegangen werden. Das im Glasrohr unter Abschlufs der Luft bei Rotglut hergestellte Produkt gab beim Erhitzen auf über $1000^{\circ}$ noch Schwefel ab, dessen Dümpfe die Schmelze aus dem Porzellanrohr schleuderten, wenn zu schnell erhitzt wurde. Nachdem das Schwefeleisen in kleinen Portionen schnell zusammen- 
geschmolzen war ${ }^{1}$, wurden diese zu einer Portion von 20 g vereinigt, die Abkühlungskurve dieser Menge bestimmt und schliefslich der Regulus analysiert. Derselbe löste sich vollkommen ohne Hinterlassung eines Rủckstandes in Salzsäure und enthielt seinem Schwefelund Eisengehalt entsprechend 98.3 \% FeS und 1.7\% Fe. Auf der betreffenden Abkühlungskurve findet sich nur bei $1234^{\circ}$ ein Kristallisationsintervall von langer Zeitdauer. Dieser Befund unterscheidet sich von den Angaben von $\mathrm{LE}$ CHateliter und ZrwaLer, welche glaubten, dafs der Schmelzpunkt des Schwefeleisens bei $950^{\circ}$ liegt, was darauf zurtickzuführen ist, dafs jene Autoren das $\mathrm{FeS}$ in einer Art erhitzten, bei der die Möglichkeit der Abdampfung von gröfseren Schwefelmengen gegeben war; hierdurch erhielten sie eine Lösung von Eisen in Schwefeleisen, deren eutektische Temperatur bei $970^{\circ}$ in der Tat nahe mit der von ihnen angegebenen Temperatur übereinstimmt.

Der Schmelzpunkt des reinen Schwefeleisens mufs noch höher als bei $1234^{\circ}$ liegen, denn die Schmelze enthielt nach ihrer Abkühlung schon $1.7 \% \mathrm{Fe}$. Mit Hilfe der Bestimmung des Beginns der Kristallisation der folgenden Schmelze mit 3.5\% Fe extrapoliert sich der Schmelzpunkt des reinen Schwefeleisens auf $1300^{\circ}$. Die mikroskopische Untersuchung des Regulus mit $1.7 \%$ Eisen ergab eine vollständig homogene Struktur der gelblichen Schlifffache. Nach dem Ätzen mit Kupfersulfatlösung wurde eine sehr feine Granulierung der nach dem Polieren homogenen Oberfläche sichtbar, die sich auf den FeS-Kristallen aller Reguli findet. Da eine sehr geringe Menge metallischen Eisens erst im Regulus mit 3.5\% Fe zu finden ist, hat man anzunehmen, dafs sich etwas metallisches Eisen im kristallisierten $\beta$-FeS zwischen $1300^{\circ}$ und $130^{\circ}$ löst. Ob bei der Umwandlung des $\beta$-FeS in $\alpha$-FeS eine Trennung dieses Eisens rom FeS stattfindet, konnte nicht sicher ermittelt werden; es wäre möglich, jene Granulierung als durch kleine Fe-Partikel hervorgerufen zu deuten.

Wie erwähnt, darf das $\mathrm{FeS}$ nicht bei Luftzutritt geschmolzen werden, denn sonst verliert dasselbe nicht unerhebliche Mengen Schwefel, da der Partialdruck desselben sich beim reinen Schwefeleisen einer Atmosphäre nähert. Da nach schnellem Erhitzen von Schwefeleisen mit uberschthssigem Schwefel auf $1400^{\circ}$ das Schwefel-

1 Die Schmelzungen wurden in Porzellanröhren von der Form eines Probierglases von $15 \mathrm{~mm}$ innerem Durchmesser im elektrisch geheirten Kohlerohr vorgenommen. 
eisen $1.7 \%$ Eisen enthält, haben wir in dieser Angabe eine angenäherte Bestimmung eines Punktes der Konzentrations-Temperaturkurve für den Partialdruck des Schwefeldampfes von einer Atmosphäre. Die Kurve $x$ y Fig. 2 deutet eine solche Kurve an, welche die Temperaturen angibt, bei denen der Partialdruck des Schwefels einer Reihe von Schmelzen eine Atmosphäre beträgt. Diese Kurve

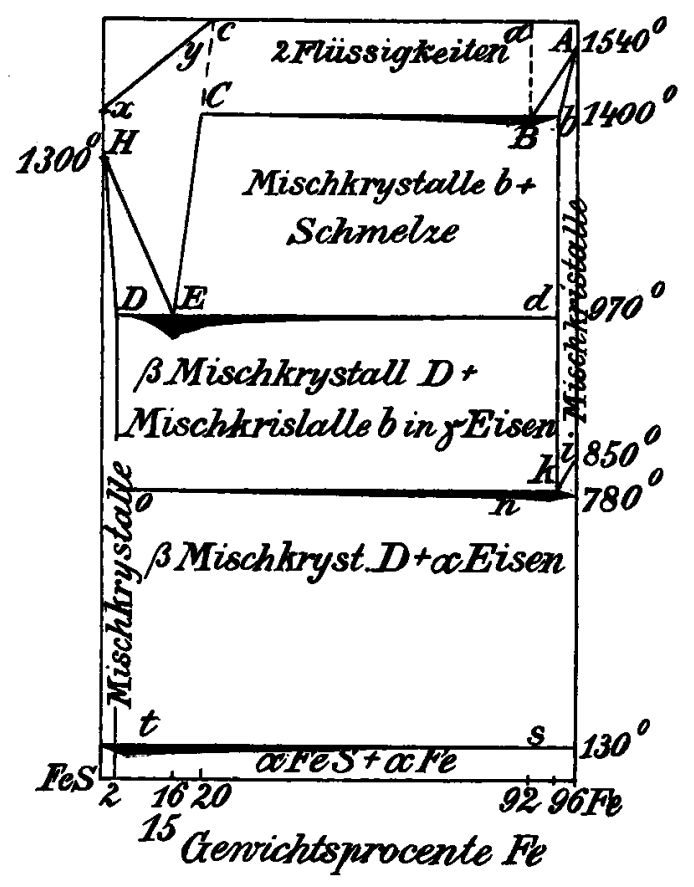

Fig. 2.

schneidet die Kurve $H E$ nicht, entsprechend der Tatsache, dafs der Partialdruck des Schwefels aus dem FeS kleiner ist als eine Atmosphäre.

Die Erscheinungen bei der Abkühlung von fiutssigen Mischungen von $\mathrm{FeS}$ und $\mathrm{Fe}$ beschreibt das Übersichtsdiagramm Fig. 2, in welches die abnormen Erscheinungen, die wahrscheinlich dem so schwer zu vermeidenden Gehalt an Oxyden zuzuschreiben sind, nicht aufgenommen worden sind. In diesem Diagramm sind die Mengen, welche bei den Temperaturen der verschiedenen nonvarianten Gleichgewichte kristallisieren, durch die Dicke der betreffenden Horizontalen angedeutet. 
Wir haben sechs verschiedene Gruppen von Schmelzen vor uns, die sich durch ihre Kristallisationswege voneinander unterscheiden. Aus den Schmelzen von 100-96\% Fe scheiden sich Mischkristalle aus, deren Kristallisation beendet ist, wenn ihre Temperatur die Linie $A b$ erreicht hat. Die Veränderungen, denen diese Gruppe von Mischkristallen unterliegt, wenn in denselben die polymorphen Umwandlungen des Eisens vor sich gehen, werden uns noch später beschäftigen.

Die Schmelzen zwischen 93 und $96 \%$ Fe hinterlassen, nachdem sich aus denselben der gesättígte Mischkristall $b$ gebildet hat, eine Schmelze von der Zusammensetzung $C$, aus der sich bei weiterer Abkihlung ebenfalls der gesättigte Mischkristall $b$ abscheidet, indem sich die Zusammensetzung der Schmelze auf der Linie $C E$ ändert. Nach Erreichung des Punktes $E$ beginnt dann die Ausscheidung des gesättigten Mischkristalles $D$ mit $3 \%$ Fe und die Schmelze kristallisiert bei $970^{\circ}$ vollständig.

Die Schmelzen zwischen 20 und $92 \% \mathrm{Fe}$ sind bei $1500^{\circ}$ zähflüssige Emulsionen zweier Flüssigkeiten, etwa von der Viskosität des Glyzerins bei $0^{0}$. Sinkt die Temperatur auf $1400^{\circ}$, so beginnt aus der eisenreichen Flussigkeit, deren Zusammensetzung dem Punkte $B$ entspricht, die Ausscheidung des gesättigten Mischkristalles $\mathrm{b}$, es zerfallt also die Flüssigkeit $B$ in den gesättigten Mischkristall $b$ und die Flüssigkeit $C$, wobei sich die Temperatur konstant erhält, bis die Flìssigkeit $B$ vollständig aufgezehrt ist. Darauf beginnt die Temperatur zu fallen, indem sich aus der Lösung $C$ der gesättigte Mischkristall $b$ ausscheidet, und fällt unter Änderung der Konzentration dieser Flüssigkeit bis zum Punkt $E$.

In den Schmelzen von $15-20 \% \mathrm{Fe}$ beginnt die Kristallisation des Mischkristalles $b$ beim Überschreiten der Linie $C E$. Die dann folgenden Erscheinungen sind die früher beschriebenen. Fig. 1, Taf. VIII zeigt die Schlifffäche eines käuflichen Schwefeleisens mit $14 \%$ metallischem Eisen. Die graue Grundmasse besteht aus FeS, dem gesättigten Mischkristall $D$, die schwarzen Flecke entsprechen Löchern und die hellen Kristalle sind metallisches Eisen.

Aus den Schmelzen von 3-15\% Fe scheiden sich zuerst Mischkristalle aus, deren Zusammensetzung sich bei fallender Temperatur auf der Linie $H D$ ändert. Nachdem sich der gesättigte Mischkristall $D$ mit $3 \% \mathrm{Fe}$ gebildet hat, beginnt die Ausscheidung des zweiten gesättigten Mischkristalles $b$ und es vollzieht sich die eutektische Kristallisation der Schmelze. Schliefslich kristallisieren die 
Schmelzen von 0-3\% Eisen als Mischkristalle, und ihre Kristallisation ist beendigt, wenn ihre Temperatur unter die Linie $H D$ gesunken ist.

Nachdem die Schmelzen vollständig kristallisiert sind, vollziehen sich in den entstandenen Kristallkonglomeraten bei der Abkühlung noch weitere Zustandsänderungen.

Auf den Abkühlungskurven der Konglomerate, welche aus käuflichem, Eisenoxyde enthaltenden Schwefeleisen hergestellt sind, finden sich bei $923^{\circ}$ deutlich ausgeprägte Haltepunkte, deren Zeitdauer mit wachsendem Eisengehalt abnimmt; die letzte Andeutung dieses Haltepunktes ist auf der Abkühlungskurve des Regulus mit 90\% Fe wahrzunehmen. Auf den Abkühlungskurven der noch eisenreicheren Schmelzen sind diese Haltepunkte nicht mehr vorhanden. Da andererseits diese Haltepunkte auf den Abkühlungskurven der FeSreichen, aber Eisenoxyde nicht enthaltenden Schmelzen nicht zu finden sind, so geht man wohl nicht fehl, wenn man ihr Auftreten mit der Bildung eines eigentiumlichen Strukturelementes von typisch lamellarer eutektischer Struktur in Zusammenhang bringt. Dieses Strukturelement findet sich nur in den oxydhaltigen Regulis. Le CHATELIER und ZIEGLER haben gezeigt, dafs die Menge dieses Strukturelementes bei Oxydation der Schmelzen wächst und andererseits bei Reduktion derselben durch Wasserstoff abnimmt.

Aufser diesen den oxydfreien Fe-FeS-Mischungen fremden Haltepunkten finden sich auf den Abkühlungskurven noch andere Haltepunkte, welche polymorphen Umwandlungen des Eisens und Schwefeleisens entsprechen.

Bekanntlich fand OsmoND ${ }^{1}$ für das reine Eisen zwei Umwandlungspunkte, bei etwa $760^{\circ}$ und $855^{\circ}$. Wir fanden diese Punkte bei $770^{\circ}$ und $850^{\circ}$. Die Umwandlungswärme bei $850^{\circ}$ scheint etwa dreimal so grofs zu sein als die bei $770^{\circ}$. Mit steigendem Gehalt an Schwefeleisen wird der Umwandlungspunkt bei $850^{\circ}$ stärker erniedrigt als der bei $770^{\circ}$, dessen Temperatur sich bei Änderung des Schwefelgehalts nicht merklich ändert. Von $90 \% \mathrm{Fe}$ an findet man auf den Abkühlungskurven nur einen Haltepunkt bei etwa $800^{\circ}$. Die Zeitdauer dieser Umwandlung pro Gewichtseinheit Eisen entspricht ungefähr der Summe jener beiden Werte beim reinen Eisen. Da der obere Umwandlungspunkt des Eisens durch Zusatz von FeS etwas verschoben wird, und da von einem Gehalt von $92 \% \mathrm{FeS}$ an

1 Contribution à l'Etude des Alliages, p. 277, Paris 1901. 
sich nur ein einziger Umwandlungspunkt auffinden läfst, so mufs man annehmen, dals sich etwas FeS in dem aus der Schmelze kristallisierenden $\gamma$-Eisen löst. Diese Annahme wird durch die früher beschriebenen Erscheinungen bei der Kristallisation der eisenreichen Schmelzen gestützt. Nur betreffs der Konzentration des gesättigten Mischkristalls bei $1300^{\circ}$ und bei $800^{\circ}$ findet man eine kleine Differenz. Bei $1300^{\circ}$ ergab sich der Fe-Gehalt im gesättigten Mischkristall $\mathrm{zu} 4 \%$, während derselbe bei $800^{\circ}$ etwa $7 \%$ entsprechend der Lage des Schnittpunktes $K$ Fig. 1 betragen würde. Da dieser Unterschied von $3 \%$ innerhalb der Versuchsfehler liegt, so ist eine Zunahme an FeS im gesättigten Mischkristall bei sinkender Temperatur nicht erwiesen.

Überschreitet bei der Abkühlung die Temperatur die Linie $i k$, so beginnt die Ausscheidung von reinem Eisen aus den Mischkristallen. Für die Ausscheidung von reinem Eisen sprechen folgende beiden Beobachtungen: Erstens ist auf den Abkühlungskurven der Reguli von $0-7 \% \mathrm{FeS}$ ein Haltepunkt vorhanden, welcher der Umwandlung von $\beta$-Eisen in $\alpha$-Eisen entspricht. Wäre bei dieser Temperatur nicht reines Eisen vorhanden, so mülste die Temperatur dieses Haltepunktes von der Umwandlungstemperatur des $\beta$ Eisens in $\alpha$-Eisen verschieden sein. Zweitens sieht man auf den Schliffen aller Reguli, dafs sich in den Eisenkristallen sehr feine Partikel von FeS befinden. Das FeS, welches bei $970^{\circ}$ kristallisiert, ist in den Zwischenräumen zwischen den einzelnen Eisenkristallen zu finden, aufserdem enthalten aber wie sowohl auf Fig. 2, Tafel VIII leider undeutlich als auch auf den zahlreichen Photogrammen von Le Chateunter und Zngalag deutlich zu sehen ist, die Eisenkristalle einen feinen Staub von FeS, der sich offenbar erst nach ihrer Kristallisation ausgeschieden hat. Dieser feine Staub von FeS müfste sich also bei der Bildung von $\beta$ - resp. $\alpha$-Eisen gebildet haben. Die Struktur der Eisenkristalle entspricht also der Bildung von $\alpha$-Eisen aus einem FeS-haltigen Mischkristall, und für die Umwandlung dieser Reihe von Mischkristallen ergibt sich aus den thermischen Erscheinungen folgendes: Ein Mischloristall von 0 bis etwa $4 \%$ FeS scheidet, wenn seine Temperatur die Linie $i k$ überschreitet, $\alpha$-Eisen aus, wodurch seine Konzentration bis auf höchstens $7 \% \mathrm{FeS}$ wachsen kann, dann zerfallt dieser Mischkristall in Schwefeleisen und $\beta$-Eisen. Im Punkte $K$ und auf der Horizontale on sind also folgende Phasen miteinander im nonvarianten Gleichgewicht: der Mischkristall mit maximalem Gehalt an Schwefeleisen, der Mischkristall 
mit maximalem Gehalt an Eisen, $\alpha$-Eisen und Dampf. Die Summe der Umwandlungswärmen von $\gamma$ - in $\beta$ und von $\beta$ - in $\alpha$-Eisen mufs also nahezu gleich sein dem Wärmeeffekt im Punkt $K$. Wie aus den in Tabelle 1 aufgeführten reduzierten. Zeiten der Umwandlungen ersichtlich ist, ist diesen Forderungen ungefähr gentugt.

Schliefslich tritt bei $128 \pm 5^{\circ}$ die Umwandlung von $\beta$-FeS in $\alpha-\mathrm{FeS}$ ein. Diese interessante Umwandlung ist schon von ' Le CHATELIFR and ZrFGLikR dilatometrisch konstatiert worden. Dieselben fanden, dafs mit sinkender Temperatur bei $140^{\circ}$ eine Kontraktion des Schwefeleisens beginnt, der dann bei $120^{\circ}$ eine starke Dilatation folgt, worauf bei $95^{\circ}$ wieder eine Kontraktion eintritt. Schliefslich ist das Volumen des FeS bei $80^{\circ}$ sehr nahe gleich dem Volumen bei $140^{\circ}$. Wir haben thermisch viel einfachere Verhältnisse konstatiert. Bei $128 \pm 5^{\circ}$ tritt auf den Abkühlungskurven bis zu $95 \% \mathrm{Fe}$ ein Haltepunkt von erheblicher Zeitdauer, welche proportional dem Gehalt an FeS zunimmt, auf. Während bei den dilatometrischen Versuchen von Le Chateungr und Ziegler die Umwandlung oder richtiger die Umwandlungen sich über ein Intervall von $60^{\circ}$ erstreckten, hatten wir es mit einem einfachen Haltepunkt zu tun. Während der Verzögerung der Ablkthhlung fiel die Temperatur nur um $10^{\circ}$. Die Umwandlung von $\beta$-FeS in $\alpha$-FeS ist sowohl von einer nicht unerheblichen Dilatation wie auch von einer nicht unerheblichen Energieänderung begleitet. Durch die Dilatation des FeS bildeten sich in den Porzellanröhren, in denen die Schmelzungen vorgenommen wurden, bei $128^{\circ}$ ziemlich weitklaffende Risse. Das Springen der Porzellanröhren bei $128^{\circ}$ trat bei allen Schmelzen von 100 bis zu 5\% FeS ein. Ein Knistern war in den Röhren während der Abkihlung schon bald nachdem die Kristallisation bei $970^{\circ}$ beendigt war, zu bemerken.

Bekanntlich wird ein Schwefelgehalt des Eisens vom Konsumenten wegen der leichten Brthchigkeit des Produktes (Kaltbruch) und vom Produzenten wegen der Schwierigkeit der Bearbeitung des Materiales (Rotbruch) gefürchtet. Die Ursachen der Verschlechterung des Eisens ergeben sich deutlich aus dem Zustandsdiagramm. Wenn ein Eisen mehr als $2.0 \%$ Schwefel $(5 \% \mathrm{FeS})$ enthält, befindet sich fast zwischen allen Eisenkörnern, aus denen das Stück zusammengesetzt ist, eine wenn auch düne Schicht Schwefeleisen, die bei $920^{\circ}$ flüssig wird. Enthält das Eisen aufserdem noch etwas Oxyde, 
so beginnt die Verflüssigung schon $100^{\circ}$ tiefer, die Eisenkörner können also bei einem Gehalt von $2 \%$ Schwefel und etwas Oxyd schon bei $800^{\circ}$ von Flüssigkeit umgeben sein und durch dieselbe aneinander haften. Fig. 3 und 4, Tafel VIII, zeigen die Struktur eines Eisens mit $92 \% \mathrm{Fe}$ und $40 \% \mathrm{Fe}$, man sieht, dafs in beiden Fällen die hellen Eisenkörner von dem dunkeln, in Wirklichkeit gelben, FeS umgeben sind. Die Folgen einer solchen Struktur auf die Zugund Bruchfestigkeit brauchen wohl nicht erst geschildert zu werden. Doch die Rotbrüchigkeit erstreckt sich noch bis $\mathrm{zu}$ einem hundertmal kleineren Schwefelgehalt, bis $0.02 \% \mathrm{~S}$, also weit in das Gebiet hinein, in dem die leicht schmelzbare Flüssigkeit zwischen den Eisenkörnern verschwindet und das Material aus Mischkristallen von $\gamma$-Fe und $\beta$-FeS besteht. Die Rotbrüchigkeit des schwefelhaltigen Eisens wird also bei kleinen Schwefelmengen von 0.02-0.2 \% Schwefel nicht durch Anwesenheit einer leichtschmelzbaren Masse zwischen den Körnern des Eisens bedingt, sondern durch die Sprödigkeit der Mischkristalle von Eisen und Schwefeleisen. Die Sprödigkeit des schwefelärmeren Eisens mülste, wie aus dem Diagramm hervorgeht, bei $850^{\circ}$ am gröfsten sein, denn bei $850^{\circ}$ bildet sich aus den spröden Mischkristallen das viel weniger spröde $\beta$ Eisen, und in der Regel nimmt die Sprödigkeit eines Kristalles mit steigender Temperatur ab. Die Tatsache, dafs als schlimmste Temperatur der Rotbrüchigkeit des Eisens Rotglut bezeichnet wird, stimmt also mit den Angaben des Diagramms überein.

Auch der Umstand, dafs die Neigung zum Rotbruch mit abnehmendem Schwefelgehalt allmählich bei etwa $0.02 \%$ verschwindet, stimmt damit uberein, dafs sich durch Beimischung von $\beta-F e S$ zum $\gamma$-Eisen eine kontinuierliche Reihe von Mischkristallen bildet, deren Sprödigkeit mit abnehmendem Schwefelgehalt auf die des reinen $\gamma$-Eisens sinkt.

Die Umwandlung des FeS unter Dilatation bestimmt die vom technischen Standpunkt minderwertigen Eigenschaften des FeShaltigen $\alpha$-Eisens bei gewöhnlicher Temperatur. Es ist leicht verständlich, dafs ein Eisen mit etwa $2.7 \% \mathrm{~S}$, also etwa $8 \% \mathrm{FeS}$, in dem die einzelnen Eisenkörner von Schwefeleisen umgeben sind, bei $130^{\circ}$ durch dessen polymorphe Umwandlung rissig wird, sich leicht pulverisieren läfst. Nimmt nun der Gehalt an $\mathbf{S}$ ab, so entsteht zwischen den einzelnen Eisenkörnern ein Zusammenhang; es entstehen Stellen festeren Zusammenhaltes und die Pulverisierbarkeit geht verloren. Doch wird die Zug- und Bruchfestigkeit noch er- 
heblich vermindert sein, weil durch die Dilatation bei $130^{\circ}$ das Gesamtgefüge gelockert ist. Erst wenn jedes FeS-Partikelchen von gröfseren Mengen Eisen umgeben ist, dürfte die Sprengkraft des $\beta$-Schwefeleisens bei seiner Umwandlung in $\alpha-\mathrm{FeS}$ nicht hinreichen, um die Eisenhülle zu sprengen oder soweit zu dehnen, um vollständig das Volumen das $\alpha$-FeS einzunehmen. Dann mufs dasselbe notwendigerweise entweder in Form von $\beta$-FeS oder einer anderen polymorphen Kristallart, die dichter als das $\beta$-FeS ist, im Eisen bei gewöhnlicher Temperatur vorhanden sein.

Das Maximum der Sprengkraft des $\beta$ - FeS haben wir - wie früher der eine von uns die des Eisens, des Jodsilbers und des Phenols ${ }^{1}$ - bestimmt. Auf eine Masse von FeS, welche das Volumen von $30 \mathrm{ccm}$ einnahm, wurde der hydrostatische Druck bei $10^{\circ}$ gesteigert. Erreichte der Druck $2900 \mathrm{Atm}$., so trat eine Umwandlung unter nicht unbedeutender Volumenverminderung ein; und wurde der Druck auf $1700 \mathrm{Atm}$. vermindert, so trat die entgegengesetzte Omwandlung ein. Hieraus folgt, dals die in einer genügend dicken Eisenhuille eingeschlossenen Partike] von FeS bei Zimmertemperatur einen Druck bis nahe an $3000 \mathrm{Atm}$. auf das sie umgebende Eisen ausüben können. Wird dieser Druck überschritten, so tritt eine Umwandlung in eine neue von der $\alpha$-Modifikation verschiedene ein, welche dann, entsprechend dem gebotenen Volumen, mit der $\alpha$-Modifikation zusammen dasselbe ausfüllt. Der Sprengdruck von ca. $3000 \mathrm{~kg}$ liegt nahe an der Elastizitätsgrenze des reinen Eisens und tief unterhalb der von Nickel- und Kohlenstoffstahlen. Es wird also ganz von dem Gehalt an Beimengungen im Eisen und von der Dicke der Eisenhülle im Vergleich zum Volumen des eingeschlossenen Schwefeleisens abhängen, ob dessen Sprengwirkung bei der Umwandlung zur Geltung kommen kann oder nicht, und ob das Schwefeleisen im Eisen bei gewöhnlicher Temperatur als $\alpha-, \beta$-FeS oder als die dichtere nur bei höheren Drucken beständige Kristallart vorhanden ist.

1 G. Tammass, Kristallisieren und Schmelzen, J. A. Barth, Leipzig 1903. Göttingen, Institut für anorgan. Chemie.

Bei der Redaktion eingegangen am 1. April 1806. 


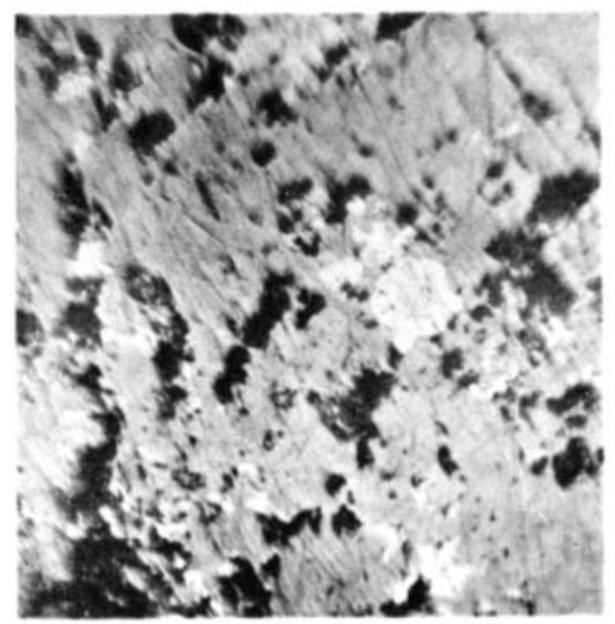

rig. 1.

$85,6 \% \mathrm{FeS}+14,4 \% \mathrm{Fe}$

(käufliches Schwefeleisen).

450 fache Vergrößerung. (Nicht geätzt.)

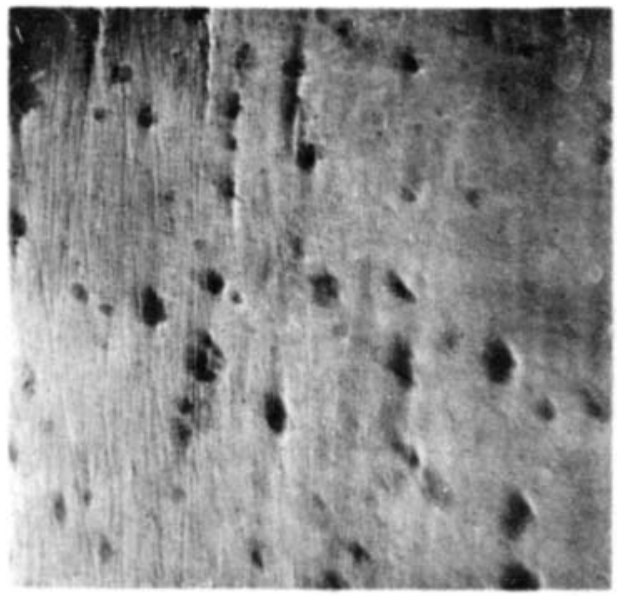

Fig. 2.

$1,9 \% \mathrm{FeS}+98,1 \% \mathrm{Fe}$.

450 fache Vergrößerung. (Nicht geätzt.)

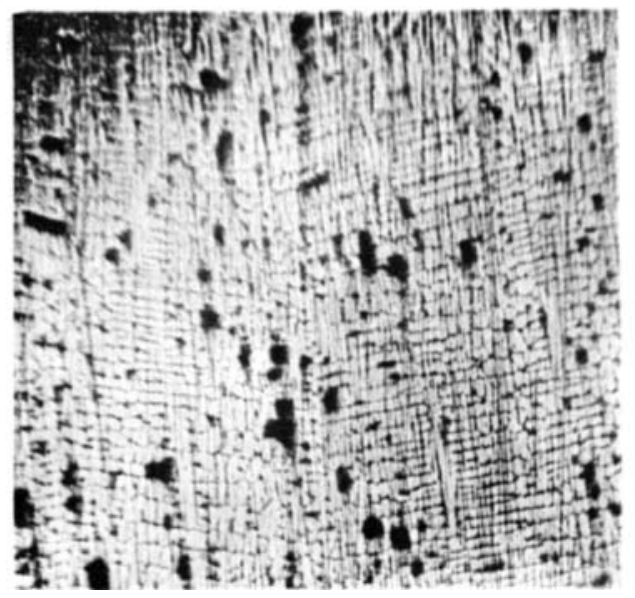

Fig. 3.

$7,9 \% \mathrm{FeS}+92,1 \% \mathrm{Fe}$.

25 fache Vergrößerung. (Nicht geätzt.)

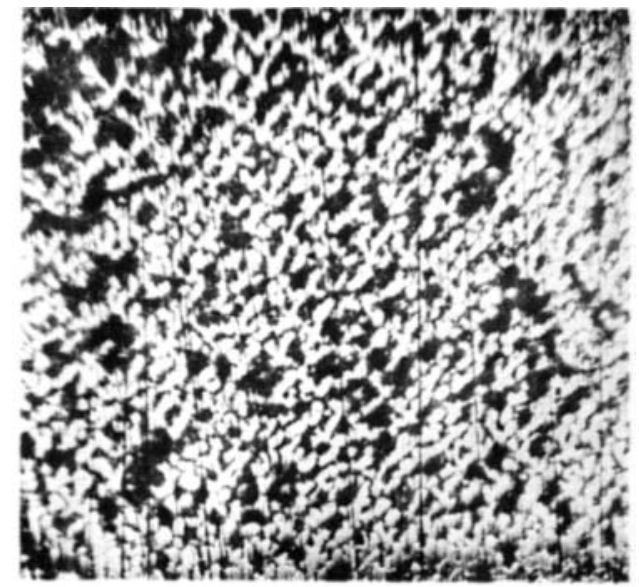

Fig. 4.

$57,7 \% \mathrm{FeS}+42,3 \% \mathrm{Fe}$.

25 fache Vergrößerung. (Nicht geätzt.) 